\title{
"Get Me Off This Medication!": A Comparison Between Gastroenterology and Primary Care Regarding Patient's Perceptions of Proton Pump Inhibitor Therapy
}

\author{
Raja Samir Khan ${ }^{1}$, Yousaf Hadi ${ }^{2}$, Swapna Gayam ${ }^{2}$ \\ 1. Internal Medicine, West Virginia University School of Medicine, Morgantown, USA 2. Digestive Diseases, West \\ Virginia University School of Medicine, Morgantown, USA
}

Corresponding author: Raja Samir Khan, rajasamirkhan.aku@gmail.com

\section{Abstract \\ Background}

Not much is known about patient perceptions regarding proton pump inhibitor (PPI) de-escalation. We sought to determine the knowledge of adverse effects (AEs) and willingness to de-escalate therapy among patients presenting to primary care and subspecialty clinics.

\section{Methods}

We conducted an anonymous survey of patients presenting to family medicine, internal medicine, and gastroenterology clinics who use PPIs. Survey topics included awareness of and concern for AEs of PPIs, and willingness to de-escalate PPI therapy.

\section{Results}

The sample comprised 206 participants presenting to the gastroenterology (29.8\%), internal medicine (32.2\%), and family medicine clinics (38\%). Of the participants, $16 \%$ were "extremely concerned" about AEs and $28.2 \%$ reported attempting to stop PPIs by themselves in the past. Many patients (54.9\%) reported that providers had not discussed AEs before initiation. Patients visiting digestive disease clinics were no more likely to report discussions on AEs and de-escalation or discontinuation attempts compared to primary care patients ( $\mathrm{p}$-values > 0.05). On logistic regression analysis, concern for AEs and counseling regarding PPI discontinuation were found to be significantly associated with attempts to discontinue PPI.

\section{Conclusions}

Although many patients on PPIs are concerned about AEs, a low number of patients reported providerinitiated discussions on AEs of PPI at initiation.

Review began 09/28/2020 Review ended 10/08/2020 Published 10/25/2020

\section{(c) Copyright 2020}

Khan et al. This is an open access article distributed under the terms of the Creative Commons Attribution License CC-BY 4.0., which permits unrestricted use, distribution, and reproduction in any medium, provided the original author and source are credited.
Categories: Family/General Practice, Internal Medicine, Gastroenterology

Keywords: proton pump inhibitor, perspectives, discontinuation, adverse effects

\section{Introduction}

Proton pump inhibitors (PPIs) have been documented extensively in the literature to be among the most commonly prescribed drug classes in the United States [1]. A growing number of studies have noted the association of PPIs with adverse effects such as Clostridium difficile infection [2], fractures [3], and acute and chronic kidney disease [4]. There has also been much discussion regarding the clinical significance of such side effects and their association with PPIs [5], with emphasis on potential discontinuation or dose reduction of these medications [6]. Recently, PPI de-escalation initiatives have been launched at various institutions [7,8]. PPIs are available over the counter and are prescribed by both primary care and specialist gastroenterologists. Per a national survey that sought to evaluate the association between concerns regarding PPI-related side effects and prior attempts to stop such medications, it was noted that concern regarding the side effects of PPIs was common and strongly associated with attempts to stop taking the medication regardless of a prescriber's recommendation [9]. Despite the attention that PPIs and their adverse effects have garnered in the literature currently, little is known about how such adverse effects are perceived by patients presenting to different subspecialties and primary care. In this study, we sought to determine the difference in knowledge of such adverse effects and willingness to de-escalate or discontinue PPI therapy between patients presenting to primary care and those presenting to subspecialty gastroenterology clinics.

\section{Materials And Methods}


We conducted a survey at clinics operated by West Virginia University. Before commencement, the study was reviewed and approved by our institutional review board. Patients more than 18 years of age presenting to family medicine, internal medicine, and gastroenterology clinics at West Virginia University were presented the study advertisement and consent at the check-in desk; they could self-administer the survey if they wished to enroll. Patients were asked to complete the survey only if they were taking a PPI. A list of generic and trade names of PPIs was included in the cover letter. They then deposited the survey in a drop box placed at the check-in desk after completing the survey.

The questionnaire was adapted and modified from a previously tested questionnaire developed by the University of Michigan [9]. The survey remained active for one month. No incentive was offered for completing the survey.

The questionnaire included multiple-choice questions on baseline demographics, use of PPIs, knowledge and perceptions regarding adverse effects of PPIs, indications for PPIs, and previous attempts and perceptions regarding PPI discontinuation.

Patients were considered at high risk of gastrointestinal (GI) bleeding if they were on dual antiplatelet therapy, if they were using anticoagulants or steroids along with anti-platelet agents or non-steroidal antiinflammatory drugs (NSAIDs), or if they were taking antiplatelet agents or NSAIDs in the setting of a history of peptic ulcer disease. A multivariable logistic regression model was applied with "any attempt at discontinuation due to adverse effect" as the dependent variable, and age, gender, specialist vs primary care clinic, whether the patient was told to discontinue PPI by their provider in the past, and the level of concern for adverse effect endorsed by the patient as covariates. All p-values less than 0.05 were considered significant. Data analysis was completed using the software SPSS Version 20 (IBM Corp., Armonk, NY, USA).

\section{Results}

\section{Population characteristics}

A total of 206 survey responses were received. Most participants were Caucasian (88.8\%), and the sample comprised a majority of females (59.7\%). The mean age of participants was 54.92 years (standard deviation: 15.74).

Of these patients, 78 patients were enrolled from family medicine clinics (38\%), whereas 66 (32.2\%) and 61 (29.8\%) patients were presenting to internal medicine and gastroenterology clinics, respectively. Omeprazole was the most common PPI among the study population (38.8\%) followed by pantoprazole (25.7\%).

Most patients were taking PPI with a diagnosis of gastroesophageal reflux disease (GERD) (87.4\%). Most patients reported that they were established with a primary care physician (PCP) (93.2\%) and believed that the PPI had been prescribed by their PCP (61.7\%); 44 patients attributed the PPI prescription to a GI specialist (21.4\%). Twelve patients did not recall the provider who prescribed the PPI (5.8\%).

Over-the-counter PPI use was reported by $18.4 \%$ of participants. Characteristics of the study population are detailed in Table 1.

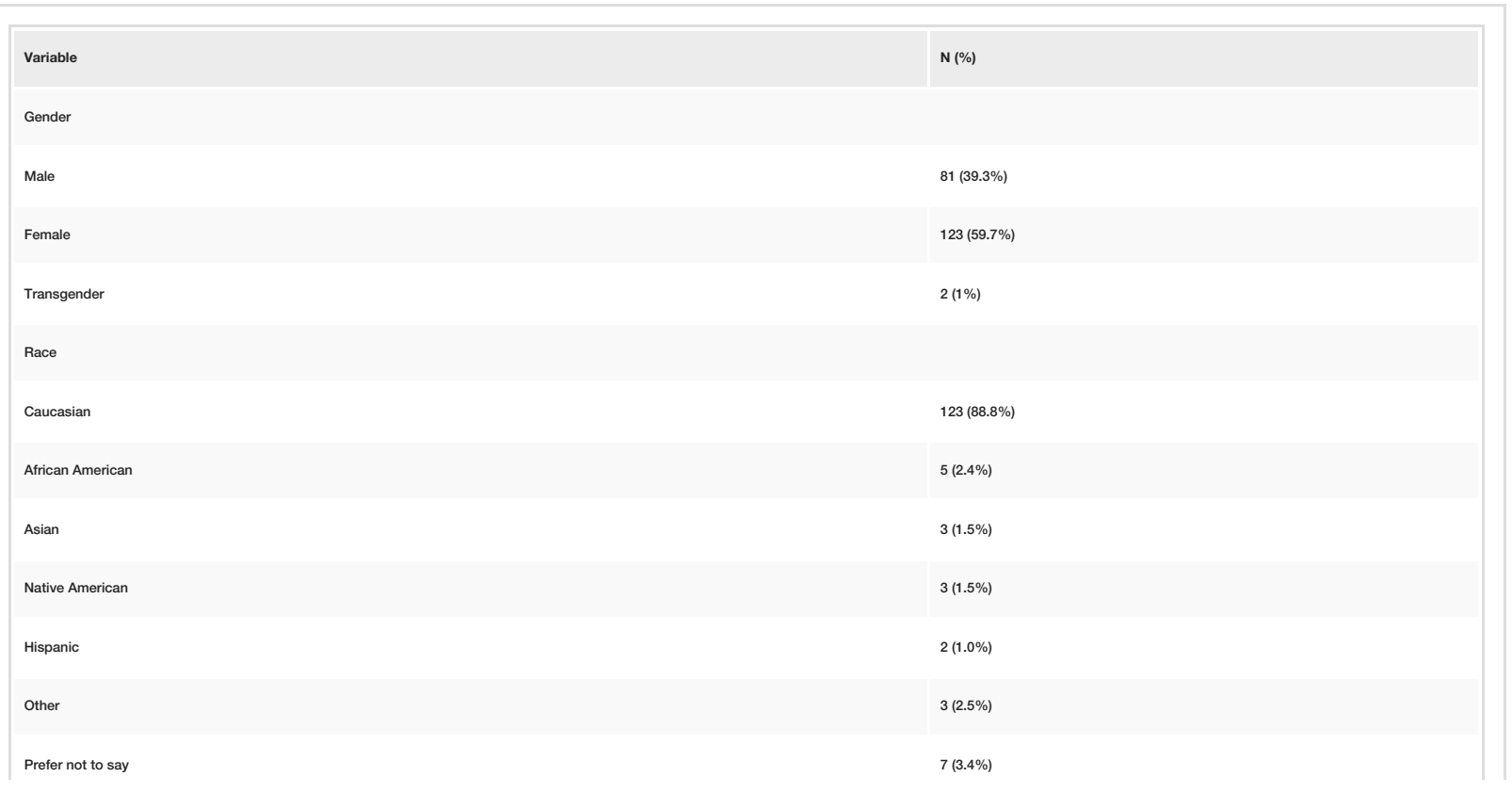




\section{Cureus}

\begin{tabular}{|c|c|}
\hline Family medicine & $78(38 \%)$ \\
\hline Internal medicine & $66(32.2 \%)$ \\
\hline Digestive diseases & $61(29.8 \%)$ \\
\hline \multicolumn{2}{|l|}{ Prescriber of PPI } \\
\hline Primary care provider & $127(61.7 \%)$ \\
\hline Digestive diseases & $44(21.4 \%)$ \\
\hline Pulmonology & $2(1.0 \%)$ \\
\hline Ear, nose, and throat & $10(4.9 \%)$ \\
\hline Others & $15(7.3 \%)$ \\
\hline Over the counter & $8(3.9 \%)$ \\
\hline \multicolumn{2}{|l|}{ Taking PPI for GERD } \\
\hline Yes & $180(87.4 \%)$ \\
\hline No & $26(12.6 \%)$ \\
\hline \multicolumn{2}{|c|}{ Non-GERD indications for PPI } \\
\hline Peptic ulcer disease & $38(18.4 \%)$ \\
\hline Esophagitis & $44(21.4 \%)$ \\
\hline Barrett's esophagus & $9(4.4 \%)$ \\
\hline History of GI bleed & $9(4.4 \%)$ \\
\hline Helicobacter pylori & $17(8.3 \%)$ \\
\hline \multicolumn{2}{|l|}{ High risk of Gl bleeding } \\
\hline Yes & $79(38.3 \%)$ \\
\hline No & $127(61.7 \%)$ \\
\hline
\end{tabular}

TABLE 1: Characteristics of the study population

PPI, proton pump inhibitor; GERD, gastroesophageal reflux disease; GI, gastrointestinal

\section{Knowledge and perspectives of adverse effects}

Of the sample, $16 \%$ and $28.6 \%$ of the participants reported being extremely concerned and somewhat concerned about adverse effects of PPIs, respectively. However, a majority of patients reported being "not at all" or "slightly" familiar with reports of adverse effects ( $36.4 \%$ and $27.7 \%$, respectively).

A minority of patients (9.2\%) reported having ever experiencing an adverse effect with PPIs. Of those who had been diagnosed with an adverse effect of PPI, only 3.8\% reported that their provider had discussed discontinuation of PPI; 58.3\% patients were "very comfortable" about discussing PPI discontinuation with their provider.

A majority of patients reported that their prescribing practitioner had not discussed adverse effects when prescribing the PPI (54.9\%), whereas $27.7 \%$ patients reported that could not recall any such conversation.

\section{Impact of PPI on quality of life}

Most patients (59.2\%) either reported no GERD symptoms or reported their severity as just noticeable, and of the total sample, $86.8 \%$ of participants reported moderate-to-complete resolution of GERD symptoms with PPI use. A majority of patients (72.3\%) reported that they could probably or definitely not manage their symptoms without PPIs. Overall, $76.7 \%$ of patients attributed PPIs to have moderate-to-high effect on their quality of life. 


\section{Cureus}

\section{Perspectives and practices regarding PPI discontinuation}

Of the participants, $28.2 \%$ reported that they had themselves tried to discontinue PPIs and $30.6 \%$ reported attempts at reducing the dose of PPIs by themselves in the past.

A minority of patients (22.8\%) reported that their health care practitioner had attempted reducing their PPI dose or attempted to discontinue their PPI completely (10.7\%).

Overall, a majority of patients rated their willingness to reduce or stop PPI favorably, with 38.8\% and 22.3\% of participants reporting that they were "somewhat willing" and "very willing" to discontinue PPI, respectively. Perspectives of patients regarding PPI discontinuation are detailed in Table 2.

Variable

Concern about adverse effects

Not at all concerned

Slightly concerned

Somewhat concerne

Extremely concerned

Yes

Attempted to reduce PPI dosage

Yes

No

HCP discussion regarding adverse effects of PPIs

Yes

Cannot recall

HCP discussion regarding dose reduction of PPIs

Yes

№

Cannot recall

HCP discussion regarding discontinuation of PPIs

Yes

No

Cannot recall

Willingness to reduce dosage of PPI

Very unwilling

Somewhat unwilling

Somewhat willing

Very willing

Willingness to stop taking PPI

\section{$\mathrm{N}(\%)$}

$45(21.8 \%)$

$69(33.5 \%)$

$59(28.6 \%)$

$33(16.0 \%)$

$58(28.2 \%)$

148 (71.8\%)

$63(30.6 \%)$

$143(69.4 \%)$

$36(17.5 \%)$

$113(54.9 \%)$

$57(27.7 \%)$

$47(22.8 \%)$

$140(68.0 \%)$

$19(9.2 \%)$

$22(10.7 \%)$

$159(77.2 \%)$

$25(12.1 \%)$

$22(10.7 \%)$

$38(18.4 \%)$

$80(38.8 \%)$

$66(32.0 \%)$

$31(15.0 \%)$

$49(23.8 \%)$ 


\section{Cureus}

\begin{tabular}{|c|c|}
\hline Somewhat willing & $80(38.8 \%)$ \\
\hline Very willing & $46(22.3 \%)$ \\
\hline \multicolumn{2}{|c|}{ Willingness to stop taking PPI if able to go back on current dose if needed } \\
\hline Very unwilling & $27(13.1 \%)$ \\
\hline Somewhat unwilling & $26(12.6 \%)$ \\
\hline Somewhat willing & $77(37.4 \%)$ \\
\hline Very willing & $76(36.9 \%)$ \\
\hline \multicolumn{2}{|c|}{ Willingness to stop taking PPI and take less powerful GERD medication } \\
\hline Very unwilling & $29(14.1 \%)$ \\
\hline Somewhat unwilling & $33(16.0 \%)$ \\
\hline Somewhat willing & $76(36.9 \%)$ \\
\hline Very willing & $68(33.0 \%)$ \\
\hline \multicolumn{2}{|c|}{ Willingness to stop taking PPI if tapered off slowly } \\
\hline Very unwilling & $20(9.7 \%)$ \\
\hline Somewhat unwilling & $20(9.7 \%)$ \\
\hline Somewhat willing & $92(44.7 \%)$ \\
\hline Very willing & $74(35.9 \%)$ \\
\hline
\end{tabular}

TABLE 2: Perspectives of patients regarding PPI adverse effects and discontinuation

PPI, proton pump inhibitor; HCP, health care provider; GERD, gastroesophageal reflux disease

Regarding strategies to discontinue PPI, participants rated all strategies favorably. They were "somewhat willing" and "very willing" to attempt discontinuation if they were asked to slowly asked to reduce dose (80.6\%), or with alternative strategies including "stop and replace with H2 blocker" (69.9\%) or "stop and be allowed to go back on current dosage if discontinuation fails” (74.3\%).

\section{Factors associated with attempts at discontinuation}

A logistic regression model was applied incorporating age, gender, specialist vs primary care clinic, whether the patient was told to discontinue PPI by their provider in the past, and the level of concern for adverse effect endorsed by the patient. Results of the regression are detailed in Table 3. Compared to patients who were "not at all concerned" about adverse effects, patients who were "somewhat" or "extremely" concerned about adverse effects of PPIs were significantly more likely to have attempted PPI discontinuation in the past. Patients who had been told to discontinue PPIs by providers were also more likely to have attempted discontinuation. There was no difference regarding attempts at discontinuation between patients who were presenting to a specialist and those presenting to primary care clinics. 


\section{Cureus}

\begin{tabular}{|c|c|c|c|}
\hline Variable & Odds Ratio & $95 \%$ Confidence Interval & p-Value \\
\hline Age & 1.002 & $0.978-1.026$ & 0.882 \\
\hline \multicolumn{4}{|l|}{ Gender } \\
\hline Male & Reference & & \\
\hline Female & 0.994 & $0.479-2.065$ & 0.988 \\
\hline Transgender & 3.789 & 0/197-73.060 & 0.378 \\
\hline \multicolumn{4}{|c|}{ Concern for adverse effects } \\
\hline Not at all concerned & Reference & & \\
\hline Slightly concerned & 2.347 & 0.678-8.131 & 0.178 \\
\hline Somewhat concemed & 6.015 & $1.760-20.562$ & 0.004 \\
\hline Extremely concerned & 13.764 & $3.752-50.494$ & 0.000 \\
\hline \multicolumn{4}{|l|}{ High risk of bleeding } \\
\hline No & Reference & & \\
\hline Yes & 1.407 & 0.668-2.964 & 0.370 \\
\hline \multicolumn{4}{|c|}{ HCP recommended discontinuation } \\
\hline No & Reference & & \\
\hline Yes & 9.129 & $3.106-26.835$ & 0.000 \\
\hline \multicolumn{4}{|l|}{ Clinic specialty } \\
\hline Primary care & Refer & & \\
\hline Digestive disease & 0.784 & $0.356-1.725$ & 0.545 \\
\hline
\end{tabular}

TABLE 3: Logistic regression analysis for factors associated with attempts at discontinuation of proton pump inhibitor therapy

HCP, health care provider

\section{Discussion}

Based on our survey, it is noted that $44.6 \%$ of the sample population endorse concerns about the adverse effects of using PPIs for GERD and 30.6\% endorse having tried to reduce the dosage or stop PPIs in the past without the recommendation of their physician (28.2\%). Per literature, it is noted that patients at high risk of upper gastrointestinal bleeding due to pharmacological and clinical risk factors benefit from ongoing PPI usage $[5,10]$. The national survey conducted recently noted that most patients using PPIs for GERD endorse concerns about adverse effects with strong association with prior attempts to stop these medications, and most patients who attempted to stop PPIs also did so without an official recommendation from their physician [9]. These findings are consistent with our survey, and when taken together, they suggest that PPI de-escalation attempts are endorsed by a minority of patients, and even in that population, most deescalation attempts are patient-led. Provider-led attempts at de-escalation were only endorsed by $10 \%$ of the population studied in our cohort. Furthermore, the finding that many patients attempt discontinuation on their own also raises some concern. This is especially important, considering that there was no difference in prior attempts to stop PPIs in patients who were at a high risk of GI bleeding compared to those who were not.

The reasoning behind attempts to discontinue PPI therapy without provider recommendation requires further study and exploration. In our study, $54.9 \%$ of the sample population endorsed not having a discussion with their provider regarding the risks and benefits of PPI therapy, and a previous study noted that $24 \%$ patients endorsed having a discussion with their provider [9].

Patients should always be advised to talk with their providers regarding the potential adverse effects of the medications they are taking before attempting to make any medication changes, even those involving medications that are available over the counter. Providers should also assume a more responsible role in 
discussing the side effects of the PPIs their patients are currently taking, the intended duration of treatment, which, per literature, is a practice that is routinely overlooked [11], and the potential risks of stopping PPIs in patients who meet clinical criteria for benefit with continuation, as well as engage in shared decisionmaking with the patients on long-term PPI therapy without an appropriate indication in order to ensure that the decision to discontinue or de-escalate the medication is an informed decision $[12,13]$.

Our survey does find that most patients presenting to clinics are willing to de-escalate PPIs, even if a large proportion of these patients attribute PPIs to a significant improvement in their quality of life. Many patients believed that they could probably or definitely not control their GERD symptoms without PPIs, but they still expressed willingness to de-escalate PPI therapy. Therefore, it appears that the major barrier to PPI de-escalation may lie at the level of the provider. This is consistent with recent literature, where electronic health record based interventions directed at physicians have led to a decrease in PPI prescription [14].

The strengths of our study include recruitment of a diverse population presenting to both primary care and specialist clinics. Previous data have focused on internet-based surveys, which may appeal certain demographics of survey takers. However, our sample may under-represent over-the counter PPI users due to the nature of our study.

\section{Conclusions}

Our study thus complements previous data and in conjunction draws the important conclusion that most PPI de-escalation attempts in the United States remain patient-led, with only a small minority of patients recalling risk-benefit discussions with their providers, and that provider recommendation was associated with a high likelihood of de-escalation attempt. Therefore, we conclude that more informed conversations with patients by providers may lead to de-escalations in patients that need it and potentially reduce inappropriate self-initiated de-escalation by patients.

\section{Additional Information \\ Disclosures}

Human subjects: Consent was obtained by all participants in this study. West Virginia University Institutional Review Board issued approval 1904538404. The West Virginia University Institutional Review Board has reviewed your submission of Exempt protocol 1904538404. Additional details regarding the review are below: - This research study was granted an exemption because the Research involves educational tests, survey procedures, interview procedures or observation of public behavior and (i) information obtained is recorded in such a manner that human subjects cannot be identified, directly or through identifiers linked to the subjects; and (ii) any disclosure of the human subjects responses outside the research could not reasonably place the subjects at risk of criminal or civil liability or be damaging to the subjects financial standing, employability, or reputation [45 CFR 46.101(2)]. All exemptions are only good for three years. If this research extends more than three years beyond the approved date, then the researcher will have to request another exemption. Animal subjects: All authors have confirmed that this study did not involve animal subjects or tissue. Conflicts of interest: In compliance with the ICMJE uniform disclosure form, all authors declare the following: Payment/services info: All authors have declared that no financial support was received from any organization for the submitted work. Financial relationships: All authors have declared that they have no financial relationships at present or within the previous three years with any organizations that might have an interest in the submitted work. Other relationships: All authors have declared that there are no other relationships or activities that could appear to have influenced the submitted work.

\section{References}

1. Kantor ED, Rehm CD, Haas JS, Chan AT, Giovannucci EL: Trends in prescription drug use among adults in the United States from 1999-2012. JAMA. 2015, 314:1818-30. 10.1001/jama.2015.13766

2. McDonald EG, Milligan J, Frenette C, Lee TC: Continuous proton pump inhibitor therapy and the associated risk of recurrent Clostridium difficile infection. JAMA Intern Med. 2015, 175:784-91. 10.1001/jamainternmed.2015.42

3. Zhou B, Huang Y, Li H, Sun W, Liu J: Proton-pump inhibitors and risk of fractures: an update meta-analysis. Osteoporos Int. 2016, 27:339-47. 10.1007/s00198-015-3365-X

4. Lazarus B, Chen Y, Wilson FP, Sang Y, Chang AR, Coresh J, Grams ME: Proton pump inhibitor use and the risk of chronic kidney disease. JAMA Intern Med. 2016, 176:238-46. 10.1001/jamainternmed.2015.7193

5. Freedberg DE, Kim LS, Yang YX: The risks and benefits of long-term use of proton pump inhibitors: expert review and best practice advice from the American Gastroenterological Association. Gastroenterology. 2017, 152:706-15. 10.1053/.gastro.2017.01.031

6. New Risk With Common PPI Heartburn Drugs . (2018). Accessed: October 12, 2020: https://www.consumerreports.org/drugs/new-risk-with-common-ppi-heartburn-drugs/.

7. Wahking RA, Steele RL, Hanners RE, Lockwood SM, Davis KW: Outcomes from a pharmacist - led proton pump inhibitor stewardship program at a single institution. Hosp Pharm. 2018, 53:59-67. $10.1177 / 0018578717747192$

8. Ziegler MJ, Freyer C, Landsburg D, et al.: Guideline implementation is effective at reducing proton pump inhibitor use in hematology-oncology units: a multidisciplinary intervention for reducing Clostridioides 


\section{Cureus}

difficile risk. Infect Control Hosp Epidemiol. 2019, 40:1294-6. 10.1017/ice.2019.238

9. Kurlander JE, Kennedy JK, Rubenstein JH, Richardson CR, Krein SL, De Vries R, Saini SD: Patients' perceptions of proton pump inhibitor risks and attempts at discontinuation: a national survey. Am J Gastroenterol. 2019, 114:244-9. 10.14309/ajg.0000000000000061

10. Bhatt DL, Scheiman J, Abraham NS, et al.: ACCF/ACG/AHA 2008 expert consensus document on reducing the gastrointestinal risks of antiplatelet therapy and NSAID use a report of the American College of Cardiology Foundation Task Force on Clinical Expert Consensus Documents. Circulation. 2008, 118:1894909. 10.1161/CIRCULATIONAHA.108.191087

11. Pasina L, Urru SA, Mandelli S, Giua C, Minghetti P, SGCP Investigators: Evidence-based and unlicensed indications for proton pump inhibitors and patients' preferences for discontinuation: a pilot study in a sample of Italian community pharmacies. J Clin Pharm Ther. 2016, 41:220-3. 10.1111/jcpt.12371

12. Batuwitage BT, Kingham JG, Morgan NE, Bartlett RL: Inappropriate prescribing of proton pump inhibitors in primary care. Postgrad Med J. 2007, 83:66-8. 10.1136/pgmj.2006.051151

13. Leri F, Ayzenberg M, Voyce SJ, Klein A, Hartz L, Smego RA Jr: Four-year trends of inappropriate proton pump inhibitor use after hospital discharge. South Med J. 2013, 106:270-3. 10.1097/SMJ.0b013e31828db01f

14. Lin D, Eke C, Cai C, Thrift AP, Shukla R: Decreasing overall and inappropriate proton pump inhibitor use: perspective from a large safety-net healthcare system. Clin Gastroenterol Hepatol. 2020, 18:763-6.

10.1016/j.cgh.2019.12.015 